\title{
Laboratório Virtual Interativo para reprodução de Experimentos de Química através de Dispositivos Móveis
}

\author{
Camila Takemoto Bertolini, Juliana Cristina Braga, Edson Pimentel, Saulo Ramos
}

Centro de Matemática, Computação e Cognição - Universidade Federal do ABC (UFABC) - Grupo de pesquisa INTERA

camilinhatbmm@hotmail.com, juliana.braga@ufabc.edu.br edson.pimentel@uabc.edu.br, srdcp@hotmail.com

\begin{abstract}
Exercise practice is a strategy widely used to support learning. This is particularly important in disciplines that have practical approaches, such as teaching chemistry. However, many factors hamper reproducing didactical laboratory experiments in chemistry such as, for example, the danger of some reagents needed for experiments and the need for at least one skilled person to assist the experiments, apart from the specific space availability itself. This paper presents iLaboratory, a tool that acts as a laboratory simulator for playing some chemistry experiments, interactively, via mobile devices.
\end{abstract}

Resumo. A realização de exercícios é uma estratégia bastante utilizada para apoiar a aprendizagem. Isso é especialmente importante em disciplinas que possuem abordagens práticas, como é o caso do ensino de química. No entanto, diversos fatores dificultam a reprodução de experimentos em laboratórios didáticos de química como, por exemplo, a periculosidade de alguns reagentes necessários para os experimentos e a necessidade de haver pelo menos uma pessoa qualificada para auxiliar durante os experimentos, além da própria disponibilidade do espaço em si. Este trabalho apresenta a ferramenta iLaboratory que atua como um simulador de laboratório para reprodução de alguns experimentos de química, de forma interativa, através de dispositivos móveis.

\section{Introdução}

A variedade de aplicações móveis tem sido explorada em larga escala nos últimos anos criando soluções científicas e profissionais. Da mesma forma, diversas pesquisas têm sido desenvolvidas para aproveitar a mobilidade dos usuários.

Segundo dados do relatório do Centro de Estudos sobre as Tecnologias da Informação e da Comunicação - CETIC.br (2013), coletados na pesquisa TIC Domicílios realizada entre setembro de 2012 e fevereiro de 2013, os brasileiros estão buscando a mobilidade e intensificando a frequência de uso dos celulares e outros dispositivos móveis, contabilizando cerca de 139 milhões de usuários de celular. $\mathrm{O}$ relatório apresenta ainda uma evolução das atividades realizadas pelo telefone celular. Por exemplo, a atividade "ouvir música" subiu de 31\% em 2011 para 47\% em 2012 e a atividade "jogar" aparece em 2012 com 29\%. Quando se observa a estratificação por faixa etária, conforme tabela 1 , nota-se que esses percentuais são ainda maiores: na 
faixa de 10 a 15 anos, $69 \%$ utilizaram o celular para jogar nos últimos três meses e na faixa de 16 a 24 anos 44\% utilizaram para acessar a internet. Isso pode indicar que a disponibilização de novas possibilidades de uso, como por exemplo, ferramentas educacionais podem ser bem recebidas, ainda mais, porque é notável a massificação dos celulares e smartphones quando se tratam de pessoas em idade escolar.

\section{Tabela 1. Atividades realizadas pelo telefone celular - Percentual sobre o total de pessoas que utilizaram telefone celular nos últimos três meses (fonte: CETIC.br, 2013)}

\begin{tabular}{|l|l|c|c|c|c|}
\hline \multicolumn{2}{|c|}{ Percentual (\%) } & $\begin{array}{c}\text { Ouvir } \\
\text { músicas }\end{array}$ & Jogar & $\begin{array}{c}\text { Assistir } \\
\text { videos }\end{array}$ & $\begin{array}{c}\text { Usar a } \\
\text { Internet }\end{array}$ \\
\hline & De 10 a 15 anos & 76 & 69 & 47 & 33 \\
\hline & De 16 a 24 anos & 76 & 50 & 46 & 44 \\
\hline \multirow{2}{*}{ FAIXA ETÁRIA } & De 25 a 34 anos & 55 & 31 & 31 & 31 \\
\cline { 2 - 7 } & De 35 a 44 anos & 39 & 17 & 18 & 18 \\
\hline & De 45 a 59 anos & 22 & 7 & 6 & 9 \\
\hline & 60 anos ou mais & 5 & 1 & 1 & 3 \\
\hline
\end{tabular}

Segundo Aoki et al (2010) os dispositivos móveis oferecem um grande potencial para aplicações educacionais. Considerando-se a mobilidade do ponto de vista do estudante, vislumbra-se que a aprendizagem pode ser transportada para qualquer lugar Por exemplo, estudantes podem fazer uma revisão para um teste enquanto estão em trânsito para algum lugar.

No âmbito internacional, Gedic et al. (2012) afirmam que os dispositivos móveis adicionam flexibilidade ao processo de aprendizagem e oferecem grandes oportunidades para a educação, especialmente para os adolescentes que demonstram grande atenção e atratividade por esses dispositivos. Martin e Ertzberger (2013) investigaram se a alta disponibilidade dos dispositivos móveis (aqui e agora) melhoram os resultados dos estudantes quando comparado com os Ambientes Virtuais de Aprendizagem (AVAs).

Fernandes et al (2012) afirmam que a maioria das iniciativas de dispositivos móveis na educação brasileira ainda são de caráter experimental e de pesquisa. Uma pesquisa nos eventos organizados pela Comissão Especial em Informática na Educação (CEIE), através do google acadêmico, retornou poucos artigos. D.Filho e Barbosa (2012) apresentam uma proposta de um conjunto de características e requisitos para ambientes de m-learning (mobile learning) a fim de flexibilizar o acesso a atividades educacionais. O trabalho de Mühlbeier et al (2012) apresenta uma aplicação de histórias em quadrinhos para dispositivos móveis na plataforma Android.

Este trabalho pressupõe que aplicações educacionais para dispositivos móveis podem ajudar a melhorar a qualidade da aprendizagem apoiando especialmente atividades de "exercitação" (realização de exercícios).

Um dos elementos bastante utilizados como estratégia para a aprendizagem é a realização de exercícios. Isso é especialmente importante em disciplinas que possuem abordagens práticas, como é o caso do ensino de química. No entanto, atualmente 
existem diversos elementos que dificultam a reprodução de experimentos em laboratórios didáticos de química, destacando-se dentre outros fatores: (a) disponibilidade dos laboratórios em horários alternativos aos das aulas; (b) necessidade de haver pelo menos uma pessoa qualificada para auxiliar durante os experimentos; (c) periculosidade de alguns reagentes necessários para fazer o experimento.

Este trabalho apresenta a ferramenta iLaboratory que atua como um simulador de laboratório para reprodução de alguns experimentos de química, de forma interativa, através de dispositivos móveis. A mesma possibilita aos estudantes reproduzir (simular) a qualquer hora ou em qualquer lugar, utilizando um smartphone, experimentos feitos num laboratório de química. Espera-se que isso possa ampliar as oportunidades de aprendizagem uma vez que os experimentos podem ser repetidos quantas vezes forem necessários.

O artigo está organizado como segue: a seção 2 apresenta a descrição dos experimentos implementados na ferramenta; a seção 3 detalha o funcionamento do Mlab em relação aos dois experimentos químicos implementados; por fim, a seção 4 apresenta as considerações finais e delineia trabalhos futuros.

\section{Descrição dos Experimentos}

Nesta seção serão descritos os experimentos implementados "Laboratório Virtual Mlab". Os mesmos estão baseados em experimentos reais realizados na disciplina "Transforamações Químicas" do curso superior de graduação da Universidade XYZ “omitido para blind review". A metodologia empregada para a realização dos ensaios foi retirada da apostila adotada pelo curso [omitido para blind review].

\subsection{Experimento 1: Teste de Chama para identificação de cátions}

Este experimento identifica o cátion que forma o sólido a ser analisado. Para realizá-lo são necessários um bico de Bunsen e o sólido a ser analisado. Utilizando uma vareta de metal, coloca-se o sólido na chama do bico de Bunsen, assim o cátion do sólido emitirá um espectro específico que é caracterizado pela mudança na coloração da chama.

A Figura 1a mostra a chama do Bico de Bunsen. A Figura 1b mostra o teste de chama para o Sulfato de Cobre e a Figura 1c mostra o teste para o Carbonato de Sódio.

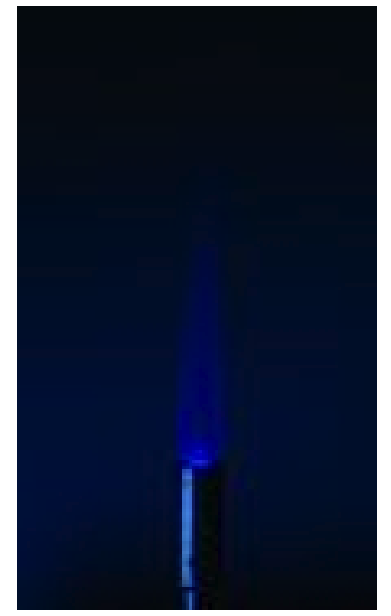

Figura 1a.

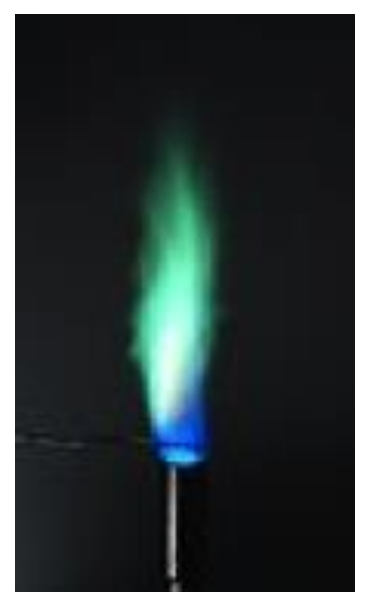

Figura 1b.

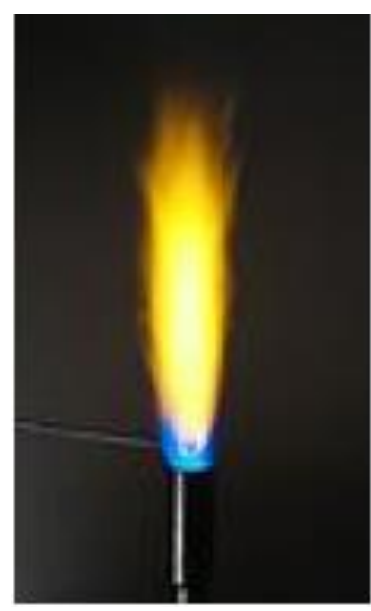

Figura 1c. 
Chama do Bico de Bunsen. Chama do Bico de Bunsen para o Sulfeto de Cobre.
Chama do Bico de Bunsen

para o Carbonato de Sódio.

\subsection{Experimento 2: Reação de Precipitação}

Neste experimento é possível identificar a solubilidade das misturas de substancias. Quando uma determinada substancia é insolúvel em outra, forma-se um precipitado sendo que este pode ser cristalino ou coloidal. Foram utilizadas três reações distintas:

\section{- Reação 1: Formação de Iodeto de Chumbo - PbI2}

Com a mistura de soluções de Iodeto de potássio - $\mathrm{KI}$ e Nitrato de Chumbo - $\mathrm{Pb}\left(\mathrm{NO}_{3}\right)_{2}$ existe a formação de Nitrato de Potássio - $\mathrm{KNO}_{3}$ que em solução aquosa é solúvel, e Iodeto de Chumbo - PbI2 que em solução aquosa é insolúvel. A equação química é dada por:

$$
2 \mathrm{KI}_{(a q)}+\mathrm{Pb}\left(\mathrm{NO}_{3}\right)_{2(a q)} \rightleftharpoons 2 \mathrm{~K}_{(a q)}^{+}+2 \mathrm{NO}_{3(a q)}^{-}+\mathrm{PbI}_{2(s)}
$$

A figura $2 \mathrm{a}$ apresenta os reagentes necessários e a figura $2 \mathrm{~b}$ mostra a aparência do precipitado, que tem uma coloração amarela do tipo coloidal.

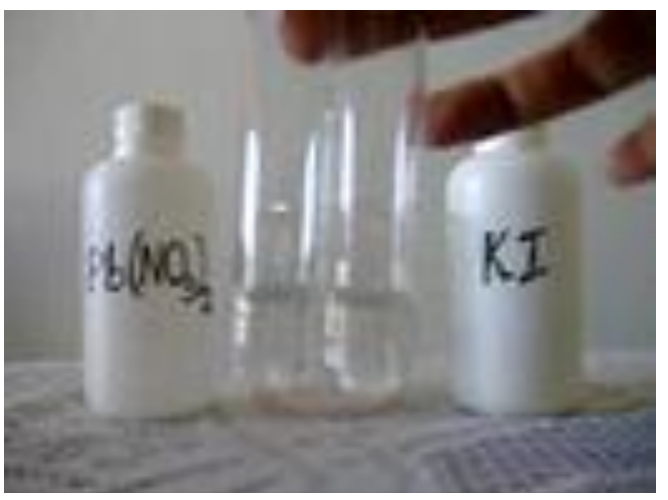

Figura 2a: Figura dos reagentes da reação de $\mathrm{Kl}$ com $\mathrm{Pb}(\mathrm{NO}) 2$

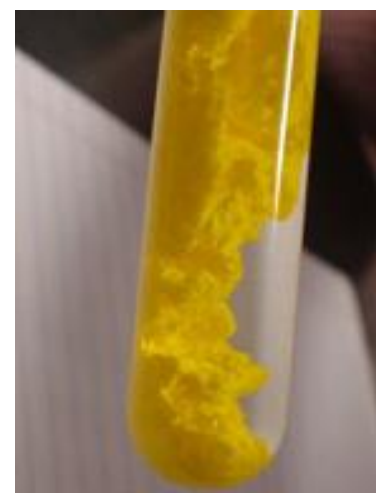

Figura 2b:

Figura do precipitado - Pb/2

\section{- Reação 2: Formação de Cloreto de Chumbo - $\mathrm{PbCl}_{2}$}

Nesse tipo de reação mistura-se o Cloreto de Potássio - $\mathrm{KCl}$ no lugar do Iodeto de Potássio. O precipitado formado é o Cloreto de Chumbo - $\mathrm{PbCl}_{2}$ e a equação química da reação é dada por:

$$
2 \mathrm{KCl}_{(a q)}+\mathrm{Pb}\left(\mathrm{NO}_{3}\right)_{2(a q)} \rightleftharpoons 2 \mathrm{~K}_{(a q)}^{+}+2 \mathrm{NO}_{3(a q)}^{-}+\mathrm{PbCl}_{2(s)}
$$
cristalino.

A figura 3 mostra o precipitado formado. Nota-se que este é branco do tipo 


\section{- Reação 3: Formação Acetato de Chumbo - Pb(H3CCOO)2}

Neste experimento utiliza-se Acetato de Potássio - $\mathrm{KCl}$ no lugar do Iodeto de Potássio e o precipitado que se forma é o Acetato de Chumbo - $\mathrm{Pb}\left(\mathrm{H}_{3} \mathrm{CCOO}\right)_{2}$. A equação química da reação é dada por:

$$
2 \mathrm{~K}\left(\mathrm{H}_{3} \mathrm{CCOO}\right)_{(a q)}+\mathrm{Pb}\left(\mathrm{NO}_{3}\right)_{2(a q)} \rightleftharpoons 2 \mathrm{~K}_{(a q)}^{+}+2 \mathrm{NO}_{3(a q)}^{-}+\mathrm{Pb}\left(\mathrm{H}_{3} \mathrm{CCOO}\right)_{2(s)}
$$

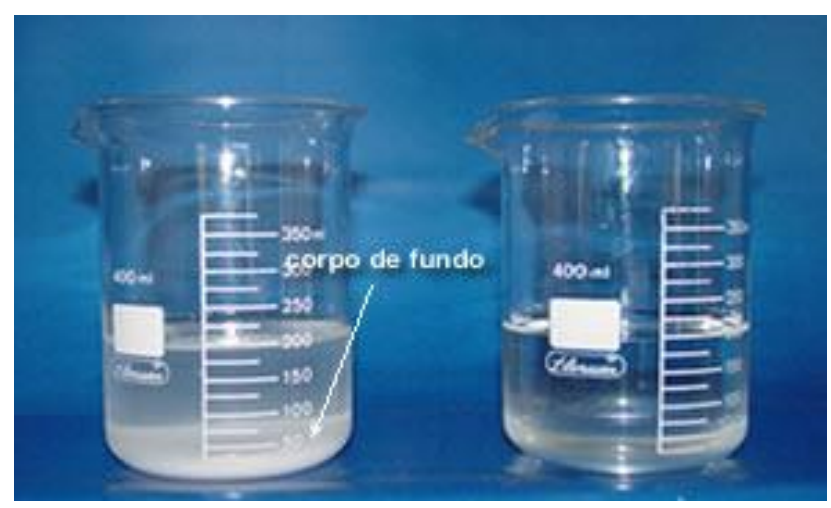

Figura 3: Formação do precipitado - $\mathrm{PbCl}_{2}$

O precipitado formado é branco do tipo cristalino e se difere pois é solúvel em água na medida de $60 \mathrm{~g} / 100 \mathrm{ml}$. A figura 4 demonstra o precipitado formado mas não em solução.

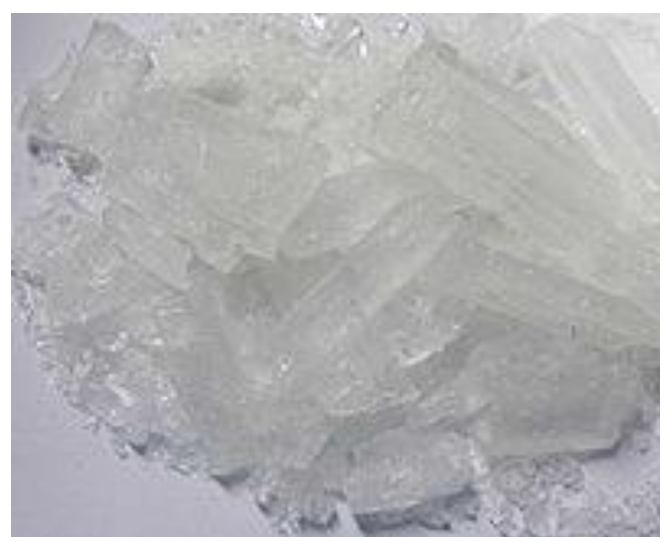

Figura 4: Formação do precipitado - $\mathrm{Pb}(\mathrm{H} 3 \mathrm{CCOO}) 2$

\section{Ferramenta iLaboratory}

O desenvolvimento do aplicativo obedeceu às seguintes etapas: (a) Determinação dos Experimentos do Projeto (descritos na seção 2); (b) Desenho das telas que compõem o aplicativo; (c) Implementação das telas básicas; (d) Implementação das telas para os experimentos.

O figura 5 apresenta a tela inicial do aplicativo iLaboratory, no aparelho Iphone, que utiliza o sistema operacional iOS5 da Apple. Para o desenvolvimento utilizou-se um equipamento Macintosh da Apple e o Ambiente de Desenvolvimento Integrado (IDE) $X$-Code, bem como a linguagem de programação Objective-C [Apple, 2012]. Na tela da figura 5 o usuário pode escolher entre os dois experimentos (descritos na seção 2) ou estudar a teoria. 
Apresenta-se a seguir os resultados das implementações dos experimentos descritos na seção 2 .

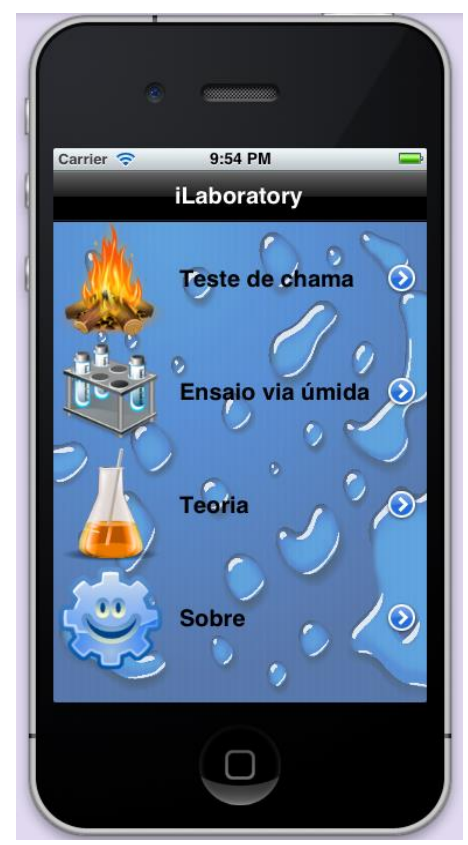

Figura 5: Menu Principal do Aplicativo

\subsection{Teste de Chama}

Neste experimento o aplicativo exibe o laboratório com o bico de Bunsen e os materiais que serão necessários para o experimento, neste caso, uma espátula para pegar o reagente e o vídro de relógio onde será colocado e reagente, conforme figura $6 \mathrm{a}$.

No canto superior direito do simulador (figura $6 \mathrm{a}$ ) pode ser ver o botão escrito "Reagentes" que quando acionado leva o usuário para a tela apresentada na figura 6b, em que o usuário pode escolher dentre uma lista, o reagente a ser utilizado na sua atividade.
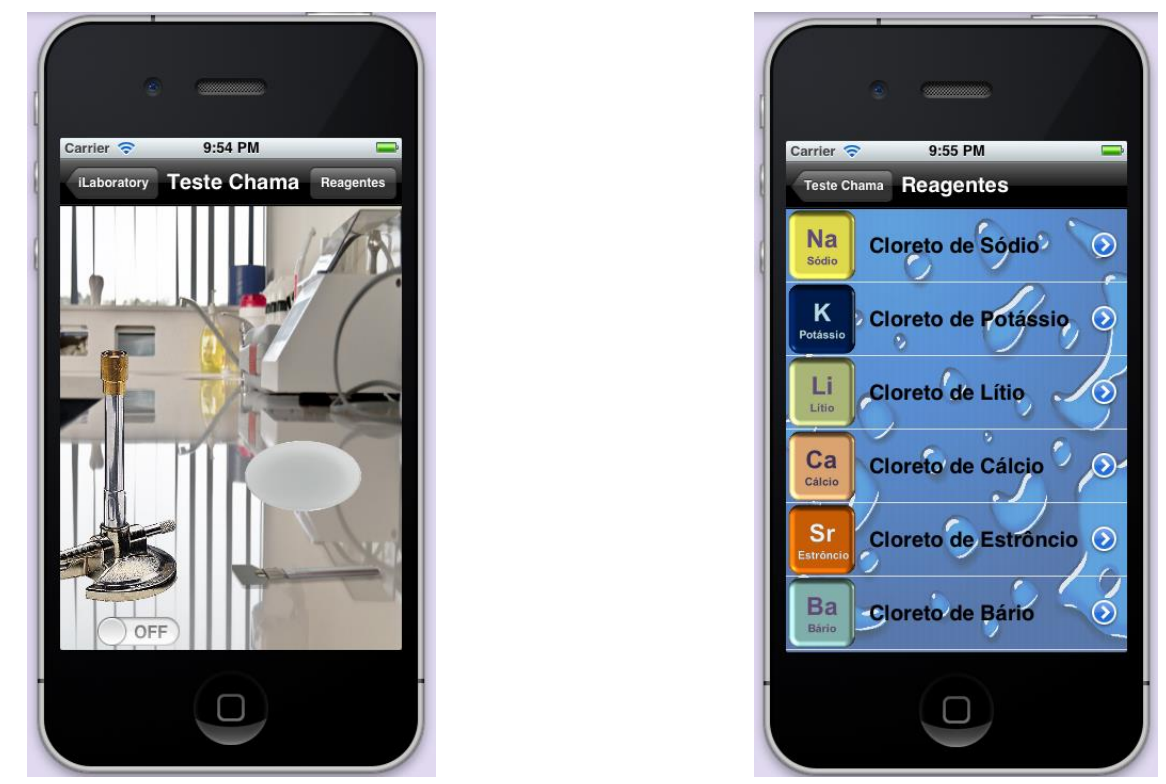
Figura 6a:

Experimento Teste de Chama.
Figura 6b:

Lista de Reagentes para o Teste de Chama.

A figura $7 \mathrm{a}$ mostra a tela que contém uma breve ficha técnica do reagente que será utilizado (escolhido na tela da figura 6b). O usuário poderá visualizar a foto do reagente e alguns dados importantes como fórmula, pontos de fusão e ebulição, coloração, entre outros dados. Caso o reagente seja o escolhido basta que o usuário pressione o botão "usar" que se encontra no canto superior direito da tela do aplicativo. Ao acioná-lo o usuário será redirecionado para a tela do laboratório, conforme figura 7b.

$\mathrm{Na}$ tela do laboratório ao se ligar o bico de Bunsen a chama aparecerá assim como o símbolo do composto para que o usuário sempre tenha uma referência do reagente utilizado, como pode ser visto na figura $7 \mathrm{~b}$. O usuário pode interagir através do “Touch Screen" levando a espátula até o vidro de relógio onde pegará o reagente e em seguida levá-la até a chama do bico que mudará sua coloração conforme o reagente selecionado. A Figura 7c mostra a mudança da coloração da chama.

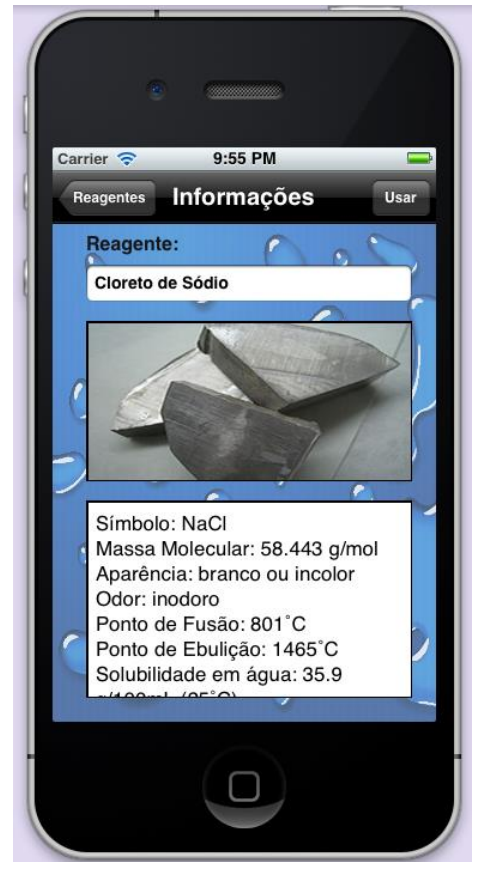

Figura 7a.

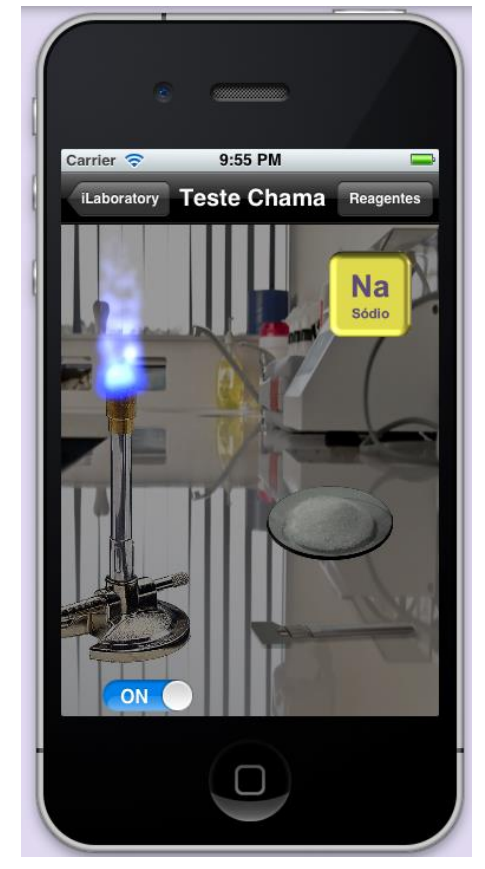

Figura $7 b$.

Ficha Técnica do Reagente. Teste de Chama com o bico de Bunsen ligado.

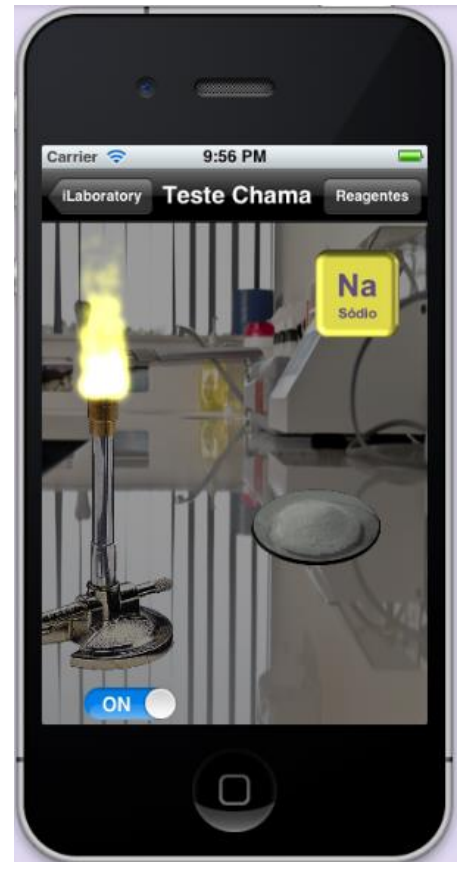

Figura 7c.

Chama reagindo com o composto.

\subsection{Ensaio por Via Úmida}

Neste experimento o aplicativo exibe o laboratório com os tubos de ensaio, o béquer, e os equipamentos que serão necessários para o experimento. $\mathrm{O}$ usuário é guiado por setas que indicam o que deve ser feito.

A figura 8a apresenta os tubos de ensaio vazios. Ao selecionar o primeiro tubo de ensaio o usuário será direcionado para a tela de escolha de reagentes conforme a figura 8b. A escolha dos reagentes segue o mesmo princípio apresentado para o Teste de Chama. O usuário escolhe o composto e em seguia é direcionado para uma tela com 
uma breve ficha técnica do reagente. A figura 8c exibe a tela com a ficha técnica em que se pode selecionar o botão "usar" ou "voltar.

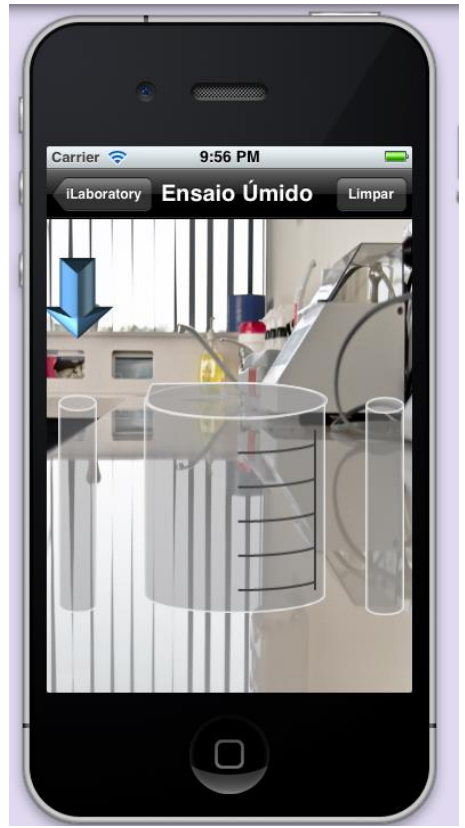

Figura 8a.

Experimento Ensaio por Via Úmida.

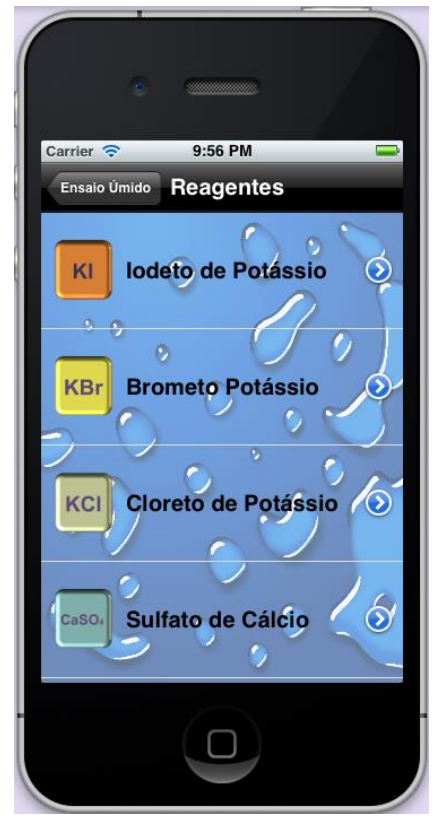

Figura 8b.

Lista de Reagentes para o primeiro tubo de ensaio.

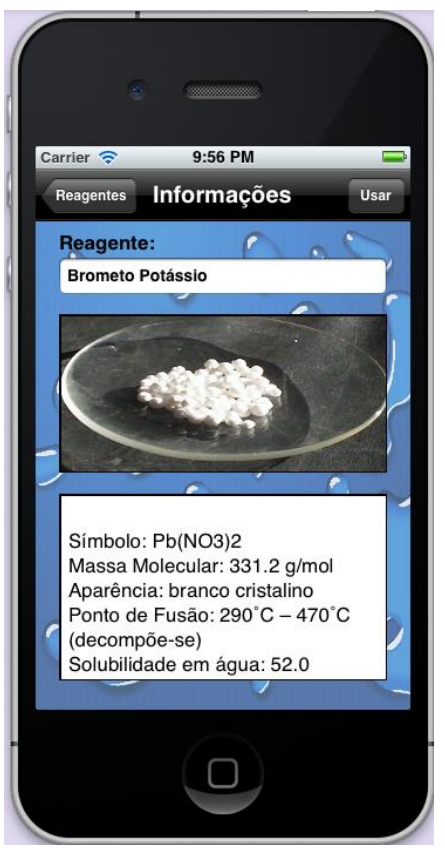

Figura 8c.

Ficha Técnica do Reagente.

Caso o reagente seja selecionado, o usuário será direcionado novamente para a tela com o laboratório e o primeiro tubo de ensaio encontrar-se-á cheio, como mostrado na figura 9a. Agora a seta que antes apontava para o primeiro tubo de ensaio aponta para o segundo tubo de ensaio (figura 9a).
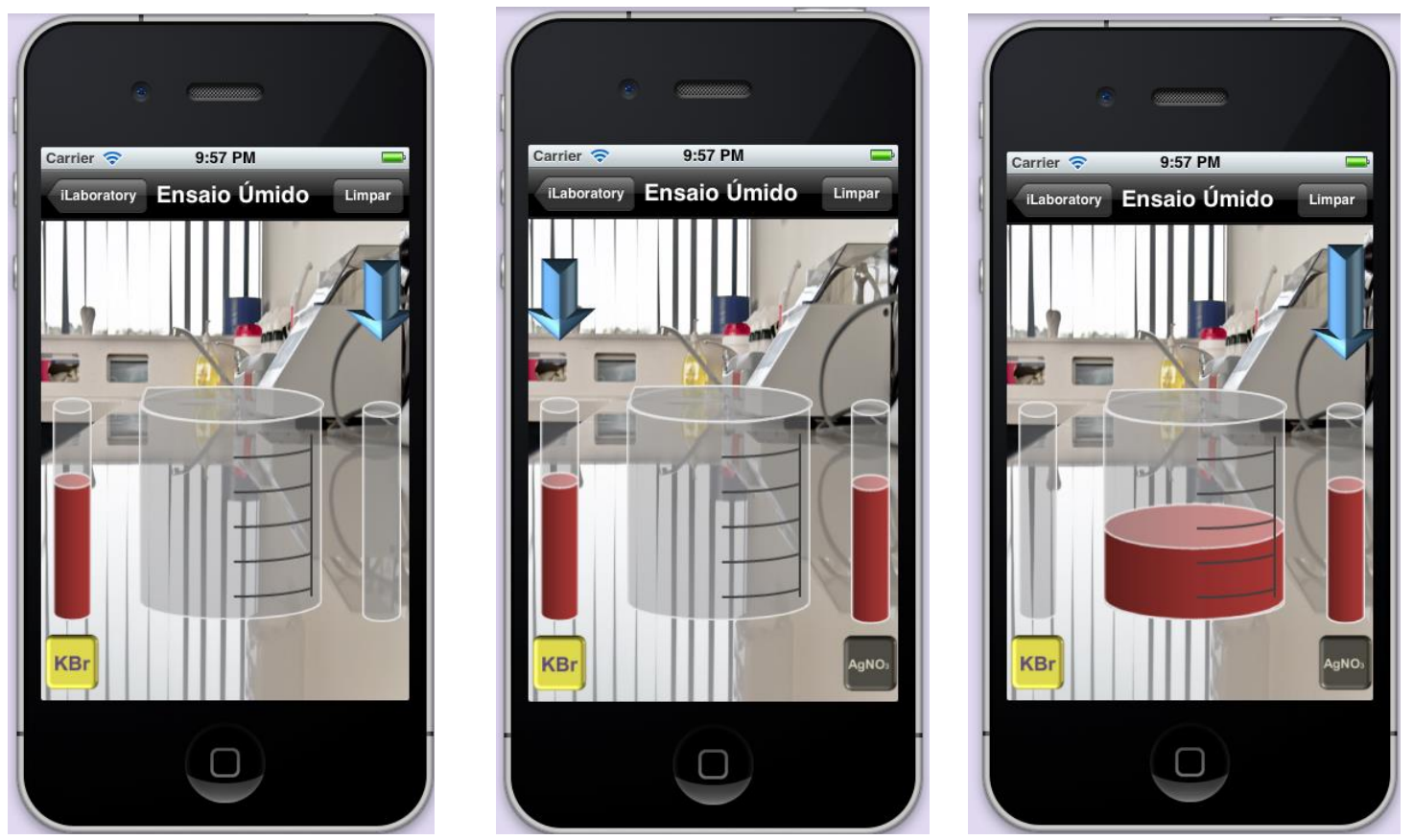
Figura 9a.

Primeiro tubo de ensaio cheio.
Figura 9b.

Primeiro e Segundo tubos de ensaio cheios.
Figura 9c.

Primeiro tubo de ensaio despejado.

O mesmo procedimento anterior deve ser realizado. Após escolhido o reagente para o segundo tubo de ensaio este se apresentará cheio na tela do laboratório como mostrado na figura 9b. Com os dois tubos de ensaio cheios a seta volta a apontar para o primeiro tubo (figura 9b). Quando o usuário selecionar o tubo o conteúdo dele será despejado no béquer conforme figura $9 \mathrm{c}$.

A Figura 10a mostra o resultado após os dois tubos de ensaio serem despejados no béquer. Para que ocorra a reação o usuário deve agitar o dispositivo móvel. A Figura $10 \mathrm{~b}$ mostra se ocorreu ou não a reação e também a equação da reação. Para recomeçar o experimento basta selecionar o botão "limpar" no canto superior direito da tela.

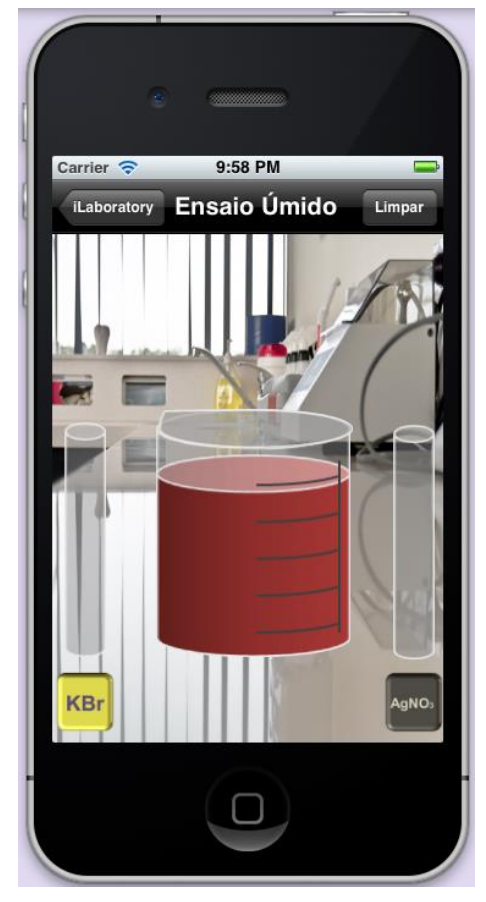

Figura 10a:

Ambos tubos de ensaio despejados

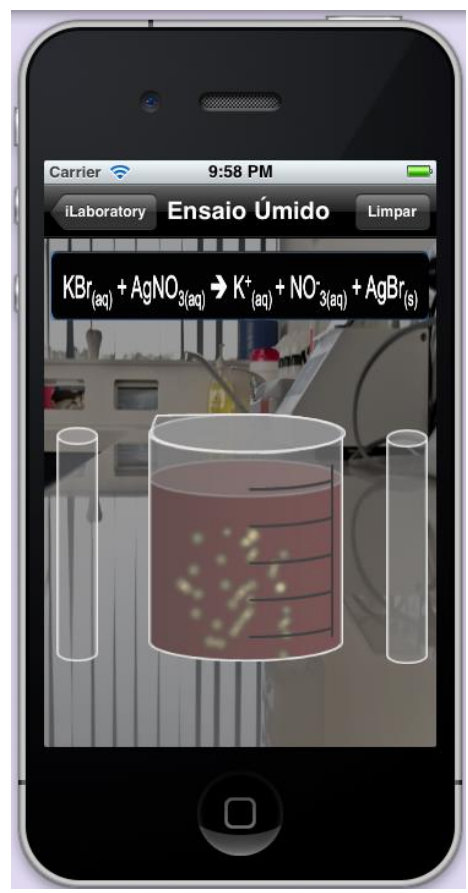

Figura 10b: Resultado Final da Reação.

O aplicativo disponibiliza ainda a opção "Teoria" que contem uma breve explicação sobre os experimentos que estão disponíveis.

\section{Considerações Finais}

Este trabalho apresentou a ferramenta iLaboratory que atua como um simulador de laboratório para reprodução de alguns experimentos de química. A ferramenta foi desenvolvida para o dispositivo Iphone da Apple e permite ao estudante interagir com diversos elementos, selecionando equipamentos (tubos de ensaios e béquer) e selecionando reagente. Na interação o estudante pode ainda conferir a ficha técnica dos reagentes e ao final do experimento selecionado, verificar e analisar o resultado, tanto de forma visual, quanto através da equação da reação. 
A ferramenta possibilita aos estudantes reproduzir (simular) a qualquer hora ou em qualquer lugar, utilizando um smartphone, experimentos feitos num laboratório de química, transpondo algumas barreiras como: (a) disponibilidade dos laboratórios em horários alternativos aos das aulas; (b) necessidade de haver pelo menos uma pessoa qualificada para auxiliar durante os experimentos; (c) periculosidade de alguns reagentes necessários para fazer o experimento. Espera-se que isso possa ampliar as oportunidades de aprendizagem uma vez que os experimentos podem ser repetidos quantas vezes forem necessários.

Como trabalhos futuros entende-se que o laboratório virtual deva ser replicado para outras plataformas, como por exemplo, o sistema operacional Android a fim de ampliar suas possibilidades de uso.

\section{Referências}

Apple. iOS Development Center, 2012. Disponível em: https://developer.apple.com/ devcenter/ios/index.action. Acesso em 11/08/2013.

CETIC.br. "Pesquisa sobre o Uso das Tecnologias de Informação e Comunicação no Brasil”. 2013. Disponível http://www.cetic.br/usuarios/tic/2012/index.htm. Acesso em 11/08/2013.

D.Filho, N. F,; Barbosa, E. F. Estudo e Definição de um Conjunto de Características e Requisitos para Ambientes de Aprendizagem Móvel. Simpósio Brasileiro de Informática na Educação. Rio de Janeiro. 2012. Disponível em http://www.brie.org/pub/index.php/sbie/ article/ view/1722. Acesso em 11/8/2013.

Fernandes, Kleber Tavares, et al. "e-Learning via Dispositivos Móveis no Brasil: Estado da Arte e Desafios à Luz do Acesso Participativo e Universal do Cidadão Brasileiro ao Conhecimento." $32^{\circ}$ Congresso da Sociedade Brasileira da Computação. Desafie! Workshop de Desafios da Computação Aplicada à Educação. 2012.

Gedik, Nuray; Hanci-Karademirci ,Arzu; Kursun, Engin; Cagiltay, Kursat. Key instructional design issues in a cellular phone-based mobile learning project, Computers \& Education, Volume 58, Issue 4, Pages 1149-1159. 2012.

Martin, Florence; Ertzberger, Jeffrey. Here and now mobile learning: An experimental study on the use of mobile technology, Computers \& Education, Volume 68, Pages 76-85. 2013.

Mühlbeier, A. R. K. et al. MOBILE HQ: O Uso de Softwares Educativos na Modalidade M-Learning. Simpósio Brasileiro de Informática na Educação. Rio de Janeiro. 2012. Disponível em http://www.br-ie.org/pub/index.php/sbie/ article/ view/1742. Acesso em 11/8/2013.

L. J. Steil and K. P. M. Frin, - Bc0307 Apostila do Laboratório de Transformações Químicas, 2011. http://www.transquim.ufabc.edu.br, 2011.

Aoki, Y; Seta, Kazuhisa; Okamoto, M (2010). Basic Consideration to Improve Self-Regulatory Skills Using Mobile Devices. ICCE2010 
II Congresso Brasileiro de Informática na Educação (CBIE 2013)

XXIV Simpósio Brasileiro de Informática na Educação (SBIE 2013) 